\title{
Armonización de las NIC/NIIF en las prácticas contables de entes emisores no financieros que cotizan en la Bolsa de Valores de Caracas, Venezuela ${ }^{1}$
}

\section{Oda Gómez}

Facultad de Ciencias Económicas y Sociales, Universidad del Zulia (LUZ)

odagomez@cantv.net

Aminta De La Hoz Facultad de Ciencias Económicas y Sociales, Universidad del Zulia (LUZ)

amihoz@yahoo.es

\section{Betty De La Hoz}

Facultad de Ciencias Económicas y Sociales, Universidad del Zulia (LUZ)

betty.hoz@hotmail.com

${ }^{1}$ Este artículo corresponde al proyecto de investigación "Proceso de aplicación de las Normas Internacionales de Información Financiera (NICNIIF) en Venezuela. Estudio empírico empresas emisoras en la Bolsa de Valores de Caracas", financiado por el Consejo de Desarrollo Científico, Humanístico y Tecnológico.

\section{Resumen}

Esta investigación de tipo documental y empírica tiene el propósito de analizar la armonización contable material de las Normas Internacionales de Contabilidad (NIC) y las Normas Internacionales de Información Financiera (NIIF) de los entes emisores no financieros; para ello, se abordó inicialmente el proceso de aplicación de las mismas en el nivel mundial, así como el proceso correspondiente en Venezuela. Se consultó el anuario estadístico de 2007 emitido por la Bolsa de Valores de Caracas; de un total de 49 empresas que aparecen en él, veinte corresponden al sector financiero y de seguros, y veintinueve al sector no financiero; de estas últimas se obtuvieron los estados financieros auditados comparativos de los años 2007-2006 de veinticinco empresas, que fue la muestra que se analiza. El análisis de los resultados indica que para la mayoría de las unidades estudiadas, en más del 70\% las prácticas de contabilidad utilizadas por las empresas objeto de estudio coinciden con las disposiciones establecidas por las NIC-NIIF. Sin embargo, se puede observar que de los 221 atributos examinados, $48 \%$ no aplica a las empresas objeto de estudio o no aparece la información en los elementos de los estados financieros; al aplicar el índice de Jaccard son excluidos de la base de datos para determinar la similitud. 
Palabras clave: armonización contable material, no financiero, Normas Internacionales de Contabilidad (NIC) Normas Internacionales de Información Financiera (NIIF).

\title{
Harmonization of IAS/IFRS accounting practices of local non-financial issuers listed on the Caracas, Venezuela stock exchange
}

\begin{abstract}
This research aims to analyze the material IAS/IFRS accounting harmonization of non- financial entities. For this purpose, we first addressed their implementation process worldwide and the corresponding process in Venezuela. Methodologically, this study is classified as a documentary and empirical research. We consulted the 2007 Statistical Yearbook issued by the Caracas Stock Exchange. Out of a total of 49 companies listed, twenty (20) relate to the financial and insurance sector, and twenty-nine (29) to the non-financial sector which were the only ones considered. For 25 of these ones comparative audited financial statements for the years 2007-2006 were obtained to form the sample under study. Analysis of the results indicates that for most of the units studied in more than $70 \%$ accounting practices used by companies under review match those provisions set by IAS-IFRS. However, we could observe that out of the 221 attributes examined $48 \%$ of them do not apply to the companies under study or no information appears in the elements of financial statements. By applying the Jaccard index they are excluded from the database to determine the similarity.
\end{abstract}

Keywords: Material accounting harmonization, non-financial, IAS / IFRS

\section{Introducción}

Las Normas Internacionales de Información Financiera (NIIF) son un conjunto de normas de alta calidad que establecen un lenguaje global y común que facilita el reconocimiento, medición y revelación de la información financiera para uso general de entidades localizadas en diferentes países.

El Consejo de Normas Internacionales de Contabilidad (NIC), es decir, el International Accounting Standards Board (IASB), es el organismo que en abril de 2001 sustituyó al Internacional Accounting Standards Comite (IASC). Actualmente, el IASB emite las NIIF, revisa y mejora las NIC. Sus actividades están comprometidas con el desarrollo de un conjunto de normas contables mundiales de alta calidad, comprensibles y de obligado cumplimiento que exijan información comparable y transparente con el fin de ayudar a los participantes en los mercados de capitales de todo el mundo y otros usuarios a tomar decisiones económicas. 
El IASB nació en 1973 con el nombre de IASC por un acuerdo establecido entre institutos profesionales de Alemania, Australia, Canadá, Francia, Holanda, Irlanda, Japón, México, Reino Unido y los Estados Unidos de América. Entre 1983 y 2001, los miembros fueron los institutos que agrupan a la profesión contable del mundo, que también eran miembros del International Federation of Accountants (IFAC). En 2001 el IASB se convirtió en un organismo independiente, una organización privada con sede en Londres, bajo la supervisión de la Internacional Accounting Standards Committee Foundation (IASCF). En el transcurso de la transformación del IASC por el IASB surgieron cambios en su estructura; el IASB dispuso adoptar como normas las emitidas por el IASC (NIC) hasta que las mismas se modifiquen o reformulen. A partir de ese momento, las nuevas normas siguieron la nomenclatura de las Normas Internacionales de Información Financiera (NIIF), que en inglés se denominan International Financial Reporting Standards (IFRS).

Durante los últimos años se ha producido un acercamiento entre los dos grandes emisores de normas de contabilidad en el nivel internacional (el IASB y la Financial Accounting Standards Board, FASB), como consecuencia de la necesidad de conseguir que las NIC-NIIF gocen de aceptación en los mercados de valores estadounidenses. Por otra parte, la FASB ha considerado conveniente codificar su extenso cuerpo de normas de contabilidad y facilitar la comparabilidad de la información financiera de las entidades que cotizan en el mercado de valores debido a que la Securities and Exchange Commission (SEC) ha aceptado las normas del IASB.

En septiembre de 2002 la FASB y el IASB acuerdan trabajar conjuntamente para acelerar la convergencia de las normas contables en el nivel mundial y celebran el "Acuerdo Norwalk" que establece dos compromisos básicos: desarrollar normas de alta calidad que sean compatibles entre sí; y eliminar una variedad de diferencias sustantivas entre NIC-NIIF y los Principios de Contabilidad Generalmente Aceptados en los Estados Unidos (USGAAP). En concreto, ambos organismos se comprometieron por realizar los máximos esfuerzos con el fin de hacer que sus normas de contabilidad vigentes resulten compatibles tan pronto como sea posible, así como coordinar sus programas de trabajo para el futuro garantizando que la compatibilidad de las normas se mantenga en el tiempo.

Este proceso de convergencia se aceleró por el requisito de la Unión Europea (UE) de aplicar las NIC-NIIF a partir de 2005. Sin embargo, la FASB y el IASB reconocen que las diferencias entre ambos cuerpos normativos son complejas de resolver, pues se requiere de tiempo. 
Dentro de los avances del mismo, en febrero de 2006 el FASB y el IASB publicaron un documento conjunto denominado Memorandum of Understanding (MoU) para agilizar el proceso de convergencia y poder lograr en el largo plazo la unificación de aquellas normas contables que ambos organismos consideren factible. Producto de este proceso de convergencia, la SEC publicó una propuesta de eliminar, a partir de enero de 2009, el requisito de reconciliación con las normas estadounidenses al que estaban sometidas todas las empresas extranjeras que presenten su formación financiera pública en Estados Unidos, cuando la información financiera sea presentada bajo NIC-NIIF.

En mayo de 2008, el FASB y el IASB publicaron los capítulos 1 y 2 del marco conceptual, que correspondía a la actualización con las observaciones recibidas a un primer documento publicado en julio de 2006, donde se proponía que el objetivo de la información financiera fuera útil para actuales y potenciales inversores de capital, los prestamistas y otros acreedores en la toma de decisiones; asimismo, se presentó una mejor descripción de las características cualitativas, entre otras, y se solicitaron comentarios hasta el 29 de septiembre de 2008. En general, parecía avanzarse a un ritmo satisfactorio.

De acuerdo con Amat et al. (2005) existe una tendencia clara hacia la convergencia de las NIC-NIIF, aunque lenta. Sin embargo, hay varios logros que se pueden destacar como son: la sugerencia efectuada en 2000 por la International Organization of Securities Commissions (IOSCO), que propuso a todos los países miembros de este organismo la utilización de las NIC-NIIF. Esta recomendación es aceptada por la Comisión Europea en 2002, quien estableció la utilización obligatoria de estas normas a partir de 2005 en los estados financieros consolidados de las compañías que cotizaban o fueran a cotizar en un mercado regulado de la UE.

Según Garza (2005), dentro de los avances hacia el progreso, China, el país más poblado de la tierra y cuyo crecimiento del producto interno bruto en los últimos años ha estado por encima del 8\%, se une a países como México, EE.UU., Japón, Singapur, entre otros, los cuales se encuentran comprometidos con la convergencia de las NIC-NIIF. En consecuencia, el 15 de febrero de 2006, el Ministro de Finanzas de China, Jin Renging, anunció la emisión de 39 normas de contabilidad para las empresas y 48 normas de auditoría para los contadores públicos certificados con el propósito de impulsar la convergencia entre las normas Chinas y las NIC-NIIF. En dicho anuncio estuvo presente David Teweedie, presidente del IASB, quien mani- 
festó que era un paso muy importante para el desarrollo de la economía de China y para su posicionamiento en los mercados internacionales.

Tomando en consideración el proceso de aplicación de las NIC-NIIF en el nivel mundial, la convergencia es como una evolución del proceso de armonización, donde se requiere una mentalidad completamente diferente con un enfoque universal, abierto y plural; puesto que se logra cuando se elige el mejor camino entre las opiniones o ideas de dos o más emisores de normas de contabilidad.

Dentro de los organismos armonizadores de las normas de contabilidad en el nivel mundial se encuentra la siguiente clasificación: 1) de carácter público, los intentos de armonización provienen de la Organización de Naciones Unidas (ONU) y de la Organización para la Cooperación y el Desarrollo Económico (OCDE); y 2) de carácter profesional, tanto la armonización de la información financiera, como de su revisión, han sido desarrollados por dos organizaciones que agrupan a profesionales de distintos países como son la IFAC y el IASB.

[...] El resultado de una serie de encuestas realizadas por firmas internacionales de contadores públicos en cincuenta y nueve países en el año 2002, revela que el $90 \%$ de los encuestados mencionan que el IASB es un organismo adecuado para desarrollar un lenguaje contable común, donde la mayoría de los países en los cuales se aplicó este instrumento han establecido mecanismos formales para lograr la armonización [...] Gómez et al. (2006)

Sin embargo, este proceso ha tomado diversas formas tales como:

- En la Unión Europea, Noruega, Rusia, Singapur y Taiwán, han sido sus gobiernos respectivos los que han establecido estrategias completas para armonizar.

- En EEUU, Australia, India, Nueva Zelanda, Sudáfrica y Hong Kong, han sido las instituciones del sector privado las que han establecido un plan formal para lograr en forma gradual dicha convergencia. En cuanto a Brasil, Uruguay, Chile, Argentina y Venezuela, han emitido principios nacionales influenciados por las NIC-NIIF.

- Otros países han evitado entrar a un proceso formal de armonización y han adoptado dichas normas, como son: Perú, Costa Rica, Honduras, Republica Dominicana, Panamá, Guatemala, Ecuador, el Salvador, Nicaragua y Haití. 
- Por otra parte, en México, el Consejo Mexicano para la Investigación y Desarrollo de Normas de Información Financiera (CINIIF) está en el proceso de comparabilidad de las normas de contabilidad mexicanas con las normas internacionales.

De acuerdo con los diferentes procesos de aplicación de las NIC-NIIF, el proceso de convergencia más importante es el desarrollado entre el IASB y FASB, donde existen preocupaciones de los observadores de este proceso por la sustancial diferencia existente entre los fundamentos sobre los que se han desarrollado los modelos de contabilidad del FASB y el IASB. Mientras el primero se ha caracterizado por la emisión de normas que contienen reglas especificas para la orientación en las prácticas de contabilidad, colocada al mero cumplimiento de las normas, más que a proporcionar información para la toma de decisiones económicas; el segundo ha emitido sus normas sobre la base de un conjunto de principios sólidos suficientes para que los encargados de elaborar la información financiera encuentren siempre las opciones que mejor representen la imagen fiel de la empresa, no proporcionando suficiente orientación y apoyo para la realización de juicios profesionales.

De la situación anterior, se deduce que las normas basadas en reglas son menos flexibles; se caracterizan por tener diferentes posibilidades para el reconocimiento de las transacciones, así como excepciones, dando un mayor detalle de aplicación a una industria especifica donde el juicio profesional se ve minimizado; mientras que las normas basadas en principios son más flexibles porque buscan la aplicación a transacciones similares sin importar la industria a la que pertenece la entidad, acepta pocas probabilidades o excepciones para evitar inconsistencias. Ahora bien, el temor que existe en la aplicación de normas basadas en principios es que la discrecionalidad conduzca a lagunas o vacíos que den origen a conductas oportunistas y a la existencia de los fraudes financieros. En tal sentido, se debe tener consciencia de que el éxito de dichas normas dependerá en gran medida del comportamiento ético de quienes elaboran la información financiera.

De acuerdo con los planteamientos ya expuestos, se puede decir que ambos entes emisores de normas de contabilidad están trabajando para hacer sus normas compatibles, elaborar normas comunes y coordinar sus programas de trabajo. Además, otras partes interesadas con gran peso, como la SEC y la Comisión de la Unión Europea, se han comprometido públicamente a colaborar para que el proceso de convergencia se complete con éxito; es decir, existen expectativas fundadas de que a mediano plazo se consiga contar con un único marco contable universal. 


\section{Proceso de aplicación de las NIC-NIIF en Venezuela}

Según Krygier (1999) dentro del marco jurídico venezolano hay varias leyes, - -incluidos el Código de Comercio, Ley del Banco Central, Ley de Impuesto sobre la Renta, Ley sobre el ejercicio de la Contaduría Pública, Ley sobre Mercado de Capitales, entre otras- que contienen disposiciones sobre el registro y presentación de la información financiera, pero ninguna de esas leyes establece principios de contabilidad; por ello, es necesario una investigación al respecto.

Dado el vacío jurídico en la emisión de principios de contabilidad en Venezuela, quien los emite es la Federación de Colegios de Contadores Públicos de Venezuela (FCCPV) ${ }^{2}$, los Principios de Contabilidad Generalmente Aceptados (PCGA) han sido admitidos por los organismos reguladores, como la Comisión Nacional de Valores (CNV), Superintendencia de Bancos y otras Instituciones Financieras (SUDEBAN), Superintendencia de Seguros y Reaseguros, Superintendencia de Empresas de Seguros (SUDESEG) y la Superintendencia de Cajas de Ahorros y Fondos de Ahorros, entre otros.

En el Directorio Nacional ampliado de la FCCPV, celebrado en Araure el 30 y 31 de enero de 2004, Estado Portuguesa, se propuso textualmente lo siguiente: "Asumir plenamente la adopción de los Principios Internacionales de Contabilidad (NICNIIF), la Normas Internacionales de Auditoria (NIA) y las Normas Internacionales de Contabilidad del Sector Gobierno (NICSP), emitidas por IFAC". Asimismo, tomando en consideración que se puedan presentar situaciones diferentes en Venezuela, se acordó proceder al estudio y evaluación de las normas de ética de la profesión y las normas de auditoría interna con estándares internacionales. Posteriormente, en Caracas, el 18 y 19 de junio de 2004, se presentó el plan de adopción ${ }^{3}$ de las normas internacionales, el cual contempla los siguientes aspectos: actualización del gremio, efecto de la información financiera y los usuarios de los estados financieros, incorporación de las universidades al cambio, cronograma para la adopción y ejecución, cualquier otra situación que genere el cambio, costo del director técnico y su equipo.

\footnotetext{
${ }^{2}$ Organismo que agrupa a los profesionales de la Contaduría Pública, que se encarga de difundir las disposiciones reglamentarias inherentes al ejercicio profesional, normas de aceptación general en la profesión, pronunciamientos inherentes a la ética del Contador Público y el régimen disciplinario. http://www.fccpv.org/ ${ }^{3}$ Publicado en la página Web de la Federación de Colegio de Contadores Públicos de Venezuela (www.fccpv.org) y divulgado en las charlas dictadas por representantes de esta institución.
} 
En cuanto a la Comisión Nacional de Valores (CNV), en diciembre de 2004 emitió la resolución No. 157-2004, indicando en su artículo 1:

Las sociedades que hagan oferta pública de valores en los términos de la Ley de Mercado de Capitales, deberán preparar y presentar sus estados financieros ajustados a las Normas Internacionales de Información Financiera (NIIF), en forma obligatoria, a partir de los ejercicios económicos que se inicien el $1^{\circ}$ de enero de 2006. En este sentido, tal preparación y presentación deberá ser efectuada mediante las Normas Internacionales de Información Financiera (NIIF) vigentes al 01 de enero de 2005.

En julio de 2005, la CNV efectuó una presentación en un evento organizado por la Cámara Venezolana Americana de Comercio e Industria (VEnAmCHAM) en el que informó que el 29 de enero de 2004 se creó el Comité Coordinador para iniciar el proceso de adopción integrado por miembros de la Comisión Nacional de Valores, empresarios y firma de contadores públicos.

Este proceso de adopción se debe al requerimiento de la IOSCO, de tal forma que todos los países que dispongan de mercado de valores presenten información homogénea debido a que los accionistas, inversores, gerentes, acreedores y el gobierno necesitan contar con información financiera confiable que les permita tomar decisiones claras y oportunas. Por mandato del comité coordinador de la CNV, el 5 de febrero de 2004 se constituye el Comité Técnico con el objeto de resolver la aplicación de los dos cuestionarios suministrados por la Comisión Nacional de Mercado de Valores (CNMV) españoles para establecer diferencias y semejanzas entre las normas locales y las NIC-NIIF. Un cuestionario se refiere a los criterios sobre reconocimiento, medición, tratamiento y registro contable de transacciones; y el otro a la información por revelar en los estados financieros y en las notas explicativas adjuntas.

Posteriormente, la CNV mediante Resolución No 177-2005 decidió posponer su aplicación hasta que la FCCPV adopte como principios de contabilidad generalmente aceptados en Venezuela las NIC-NIIF; no obstante, es permitida la adopción de las mismas por anticipado, una vez que se cumplan ciertos requerimientos ante este organismo. Ante esta situación, se deduce que el proceso de adopción de estándares internacionales, impulsado en los años 2004 y 2005 por la CNV, queda rezagado porque hasta la fecha no se ha pronunciado oficialmente sobre la entrada en vigencia de la NIC-NIIF para las empresas que cotizan en la Bolsa de Valores de Caracas. 
Una vez enunciadas las consideraciones de la FCCPV y la CNV se puede inferir que todo este proceso viene liderizado por la necesidad que tienen las empresas que cotizan en bolsa de valores de presentar la información financiera de acuerdo con las NIC-NIIF para poder competir en los mercados internacionales. En el caso de Venezuela, el mercado bursátil de acciones es incipiente de acuerdo con la Bolsa de Valores de Caracas (2007), el índice bursátil ${ }^{4}$ (IBC) finalizó en 2007 con una disminución de $27.43 \%$ con relación al cierre de 2006 después de haber experimentado en ese año con respecto a 2005 un incremento del $150 \%$.

En este orden de ideas, las entrevistas realizadas a especialistas en el mercado bursátil expresan una visión negativa acerca del crecimiento del mercado bursátil local, pues se asevera que las operaciones y las cantidades de recursos que se manejan en la bolsa son pequeñas en comparación con otras plazas y se insiste en que mientras se mantenga un panorama de incertidumbre en el aspecto económico del país, acompañado de las nacionalizaciones de las empresas que eran líderes dentro de la Bolsa de Valores de Caracas, como la CANTV y la Electricidad de Caracas, será difícil alcanzar el crecimiento observado en los ejercicios fiscales anteriores (2006-2005).

Esta investigación hace referencia a los PCGA en Venezuela, emitidos por la FCCPV, que se aplican las empresas que cotizan en la Bolsa de Valores de Caracas. Cabe aclarar, y haciendo una reseña histórica de los PCGA en este país, que en 1974 se emitió la Publicación Técnica No 3 (PT-3) con la finalidad de dar una base uniforme a la información financiera que presentan las empresas y relacionada con las normas básicas y principios de contabilidad de aceptación general. En dicha normativa se establecía que mientras este gremio no manifestara pronunciamientos sobre los principios de contabilidad en el nivel nacional se tomarían en cuenta los principios de contabilidad aceptados en México, considerando la analogía del desarrollo histórico, cultural y económico de Venezuela con México y por el reconocimiento que se tiene al Instituto Mexicano de Contadores Públicos (IMCP).

Después de más de dos décadas, la FCCPV divulgó la Declaración de Principios de Contabilidad $\mathrm{N}^{\circ} 0$ (DPC-0) referente a las normas básicas y principios de contabilidad de aceptación general y, debido a la importancia, la trascendencia que han

${ }^{4}$ El IBC está constituido por las 17 empresas de mayor capitalización en la bolsa de Caracas. Se calcula por la metodología de ponderación por capitalización de mercado. 
tenido las normas internacionales de contabilidad (NIC) y la aceptación creciente en el ámbito mundial de los negocios; así, se modifica el criterio para la aplicación supletoria de otros principios contables, quedando la jerarquía de la aplicación de los mismos en Venezuela de la siguiente forma: a) Principios publicados por la Federación de Colegio de Contadores Públicos de Venezuela (DPC), b) Normas Internacionales de Contabilidad (NIC), c) Principios de Contabilidad emitidos en México por el Instituto Mexicano de Contadores Públicos, d) Pronunciamientos Contables del Instituto Americano de Contadores Públicos (FASB) y e) PCGA de países latinoamericanos con situaciones económicas similares a las de Venezuela. Como se puede observar, la diferencia más importante con respecto a la $\mathrm{PT} \mathrm{N}^{\mathrm{o}} 3$, radica en la inclusión de las NIC, lo cual indica que desde marzo de 1997 se deben estar aplicando en Venezuela las normas indicadas en el cuadro 1 porque no existen pronunciamientos en el nivel nacional sobre el reconocimiento, medición y revelación de los elementos de los estados financieros indicados en dichas normas $\mathrm{y}$, por efecto supletorio, se deben aplicar las mismas.

Posteriormente, en 2008 se aprobó el Boletín de Aplicación No. 0 (BA VEN NIF $\mathrm{N}^{\circ} 0$ ), referente al marco de adopción de las NIC-NIIF, que señala que los ejercicios económicos que se inicien a partir del 1 de enero de 2008, y únicamente para la aplicación de las grandes entidades, estarán derogadas todas las DPC y PT relacionadas con aquéllas y en su lugar se ratifica la obligatoriedad de aplicación de las VEN-NIF y los boletines de aplicación (BA VEN-NIF) aprobados por la FCCPV.

Para las pequeñas y medianas empresas (Pyme) la derogatoria indicada en la BA VEN-NIF $\mathrm{N}^{\circ} 0$ (versión 4) será aplicada para los ejercicios que se inicien a partir del 1 de enero de 2011. También se indica que la aplicación en Venezuela de toda NIC-NIIF, o de alguna modificación emitida por el IASB, estará previamente sujeta al análisis técnico con respecto a los impactos de aplicabilidad en el entorno económico por parte del Comité Permanente de Principios de Contabilidad de la FCCPV, cuyas conclusiones serán sometidas a la aprobación en un directorio nacional ampliado.

Hasta junio de 2008 la FCCPV aprobó la aplicación en Venezuela del marco conceptual y las NIC-1, NIC-2, NIC-7, NIC-8, NIC-10, NIC-11, NIC-12, NIC-14, NIC-16, NIC-17, NIC-18, NIC-19, NIC-20, NIC-21, NIC-23, NIC-24, NIC-26, NIC-27, NIC-29, NIC-31, NIC-32, NIC-33, NIC-34, NIC-36, NIC-37, NIC-38, NIC-40, NIC-41, NIIF-1, NIIF-2, NIIF-3, NIIF-4, NIIF-5, NIIF-6 y NIIF-7; así como las interpretaciones de las NIIF (CINIIF). 


\section{Cuadro 1}

\section{NIC-NIIF vigentes en Venezuela (de marzo de 1997 a diciembre de 2007 para las grandes entidades)}

\begin{tabular}{|c|c|c|}
\hline $\begin{array}{l}\text { NIC/ } \\
\text { NIIF }\end{array}$ & Descripción & Vigencia según IASB \\
\hline NIC -1 & $\begin{array}{l}\text { Presentación de estados } \\
\text { financieros }\end{array}$ & Aprobada en 1975, última revisión en diciembre 2004 \\
\hline NIC -2 & Inventarios & Aprobada en 1975, última revisión en diciembre 2004 \\
\hline NIC -10 & $\begin{array}{l}\text { Hechos ocurridos después de } \\
\text { la fecha del balance }\end{array}$ & Aprobada en 1977, última revisión 1999. \\
\hline NIC -14 & $\begin{array}{l}\text { Información financiera por } \\
\text { segmentos }\end{array}$ & Aprobada 1981, revisada 1997 y vigencia 1998. \\
\hline NIC -16 & Propiedad, Planta y Equipo & Aprobada 1982, revisiones en 1997 y 1998. \\
\hline NIC -18 & Ingresos ordinarios & Aprobada 1982, sustituida 1993 vigente 1995. \\
\hline NIC -19 & Beneficios a los empleados & Aprobada 1983, reformada 1995, y vigente 1999 \\
\hline NIC -20 & $\begin{array}{l}\text { Contabilización de las } \\
\text { subvenciones del gobierno e } \\
\text { información a revelar sobre } \\
\text { ayudas gubernamentales }\end{array}$ & Aprobada 1983 y reformada 1994 \\
\hline NIC -24 & $\begin{array}{l}\text { Información a revelar sobre } \\
\text { partes relacionadas }\end{array}$ & Aprobada 1984 reformada 1994, revisado en el 2004 \\
\hline NIC -26 & $\begin{array}{l}\text { Contabilización e } \\
\text { información financie-ra sobre } \\
\text { planes de beneficio por retiros }\end{array}$ & Aprobada 1989 reformada 1994. \\
\hline NIC -33 & Ganancia por acción & Aprobada 1997, vigencia 1998 y revisada en el 2004 \\
\hline NIC -34 & $\begin{array}{l}\text { Información financiera } \\
\text { intermedia }\end{array}$ & Aprobada 1998, vigencia 1999. \\
\hline NIC -38 & Activos intangibles & Aprobada 1998. Vigencia 1999, revisada en el 2004 \\
\hline NIC -40 & Propiedades de inversión & Aprobada 2.000 Vigencia 2001 \\
\hline NIC -41 & Agricultura & Aprobada 2001. Vigencia 2003 \\
\hline NIIF -1 & $\begin{array}{l}\text { Adopción por primera vez de } \\
\text { las normas internacionales de } \\
\text { información financiera }\end{array}$ & $\begin{array}{l}\text { Vigente para los periodos que terminen el } 31 \text { de } \\
\text { diciembre de } 2005\end{array}$ \\
\hline NIIF -2 & Pagos basados en acciones & 1 de enero de 2005 \\
\hline NIIF -3 & Combinación de negocios & 1 de enero de 2005 \\
\hline NIIF -4 & Contratos de seguros & 1 de enero de 2005 \\
\hline NIIF -5 & $\begin{array}{l}\text { Activos no corrientes } \\
\text { mantenidos para la venta y } \\
\text { operaciones discontinuadas }\end{array}$ & 1 de enero de 2005 \\
\hline NIIF -6 & $\begin{array}{l}\text { Exploración y evaluación de } \\
\text { recursos minerales }\end{array}$ & 1 de enero de 2006 \\
\hline NIIF -7 & $\begin{array}{l}\text { Instrumentos financieros, } \\
\text { información a revelaciones }\end{array}$ & 1 de enero de 2007 \\
\hline NIIF -8 & $\begin{array}{l}\text { Información financiera por } \\
\text { segmentos }\end{array}$ & 1 de enero de 2009 , se puede adoptar con anticipación \\
\hline
\end{tabular}

Fuente: Gómez, De La Hoz y De La Hoz con base en información de IASB (2006) 
El impacto más importante en la aplicación de las normas vigentes es la sustitución del concepto de costo histórico por el de valor razonable ${ }^{5}$, lo cual podrá tener su efecto en los resultados y patrimonios de las empresas; por ello, es necesario que las mismas aumenten la cantidad, calidad, transparencia y relevancia de la información financiera, necesaria para la toma de decisiones.

Otro aspecto es el referente a la propiedad, planta y equipo que exige que todos los años se revise el método de depreciación y la vida útil de los activos, así como su deterioro. En forma general, estas normas requieren que se suministre suficiente información cualitativa sobre los elementos de los estados financieros.

\section{Aspectos metodológicos del estudio}

Nuestro estudio empírico se enmarca dentro de la armonización material porque es una comparación entre las prácticas indicadas por el IASB y las aplicadas por las empresas emisoras no financieras que cotizan en la Bolsa de Valores de Caracas, por lo que se considera importante incluir un resumen de estudios empíricos realizados en otros países sobre la armonización material (ver cuadro 2) porque no se encontraron estudios a nivel local.

Nuestra investigación es de tipo descriptiva porque se describe y analiza las características principales del problema investigado. Particularmente, se recolectaron datos referidos al reconocimiento, medición y revelación de las categorías contables seleccionadas con base en los estados financieros de las empresas objeto de estudio.

Fue necesario para su estudio determinar las unidades de los elementos de los estados financieros seleccionados, que son las cuentas que integran el activo, pasivo y patrimonio neto. Con base en el estudio de las NIC-NIIF aprobadas por la FCCPV en 2008 y el trabajo de investigación de Silva (2004) se elaboraron doce unidades de investigación (C1, C2, C3, C4, C5,....C12), las cuales se presentan en el cuadro 3, que comprenden los elementos de los estados financieros (activo, pasivo y patrimonio neto) analizados con el propósito de medir las similitudes y distancias entre la normatividad contable aplicada por las empresas objeto de estudio y la del IASB.

${ }^{5}$ Es el precio en que un comprador y un vendedor bien informados están de acuerdo para una transacción de intercambio. 


\section{Cuadro 2}

\section{Estudios sobre armonización material}

\begin{tabular}{|c|c|c|c|c|}
\hline Año & Autores & Alcance & Metodología & Conclusiones \\
\hline 1984 & Gray et al. & $\begin{array}{l}\text { Estudio de las } \\
\text { normas y prácticas } \\
\text { en } 30 \text { países. }\end{array}$ & $\begin{array}{l}\text { Clasificación del } \\
\text { tratamiento contable. } \\
\text { Cálculo del } \\
\text { porcentaje de } \\
\text { empresas que adopta } \\
\text { determinada práctica. }\end{array}$ & $\begin{array}{l}\text { No presenta } \\
\text { conclusiones, sin } \\
\text { embargo se pueden } \\
\text { analizar los resultados } \\
\text { por país, o por área } \\
\text { contable. }\end{array}$ \\
\hline 1988 & Van der Tas & $\begin{array}{l}\text { Estudio del grado de } \\
\text { armonización de las } \\
\text { prácticas contables } \\
\text { en el Reino Unido, } \\
\text { en Holanda y en los } \\
\text { EE.UU. }\end{array}$ & $\begin{array}{l}\text { Índice } \mathrm{H} \text {, } \\
\text { Índice } \mathrm{C} \text { e } \\
\text { Índice I }\end{array}$ & $\begin{array}{l}\text { Distinción entre } \\
\text { armonización formal } \\
\text { y material, y entre } \\
\text { prácticas de divulgación } \\
\text { y prácticas de } \\
\text { valoración. }\end{array}$ \\
\hline 1990 & $\begin{array}{l}\text { Tay y } \\
\text { Parker }\end{array}$ & $\begin{array}{l}\text { Análisis de seis } \\
\text { estudios sobre la } \\
\text { medida del grado de } \\
\text { Armonización. }\end{array}$ & $\begin{array}{l}\text { Se recomienda la } \\
\text { adopción del test del } \\
\text { c2 y de índice de } \\
\text { concentración }\end{array}$ & $\begin{array}{l}\text { Ninguno de los seis } \\
\text { estudios presenta una } \\
\text { verdadera medida del } \\
\text { grado de armonización } \\
\text { material. }\end{array}$ \\
\hline 1992 & $\begin{array}{l}\text { Van der } \\
\text { Tas }\end{array}$ & $\begin{array}{l}\text { Análisis del grado de } \\
\text { armonización de los } \\
\text { impuestos diferidos } \\
\text { en } 154 \text { empresas } \\
\text { europeas cotizadas } \\
\text { entre } 1978 \text { y } 1988 \text {. }\end{array}$ & Índice $C$ & $\begin{array}{l}\text { La Cuarta Directiva } \\
\text { de la UE tuvo impacto } \\
\text { positivo en las cuentas } \\
\text { individuales, en las } \\
\text { cuentas consolidadas. } \\
\text { Este impacto no es tan } \\
\text { significativo. }\end{array}$ \\
\hline 1992 & $\begin{array}{l}\text { Emenyonu } \\
\text { y Gray }\end{array}$ & $\begin{array}{l}\text { Estudio de las } \\
\text { prácticas contables } \\
\text { en Francia, Alemania } \\
\text { y Reino Unido. }\end{array}$ & $\begin{array}{l}\text { Índice I de Van der } \\
\text { Tas y el test del c } 2 \text {. }\end{array}$ & $\begin{array}{l}\text { Existían diferencias } \\
\text { significativas en las } \\
\text { prácticas de valoración. }\end{array}$ \\
\hline 1995 & $\begin{array}{l}\text { Archer, } \\
\text { Devaille y } \\
\text { McLeay }\end{array}$ & $\begin{array}{l}\text { Estudio de las } \\
\text { políticas contables de } \\
\text { empresas europeas } \\
\text { con capitales } \\
\text { extranjeros. }\end{array}$ & $\begin{array}{l}\text { Descomposición del } \\
\text { índice C de Van der } \\
\text { Tas, en dos índices. }\end{array}$ & $\begin{array}{l}\text { Se observan pocos } \\
\text { progresos en el proceso } \\
\text { de armonización en los } \\
\text { períodos de } 1986 / 87 \text { y } \\
1990-1991 .\end{array}$ \\
\hline 1995 & $\begin{array}{l}\text { Herrmann } \\
\text { y Thomas }\end{array}$ & $\begin{array}{l}\text { Estudio de las } \\
\text { prácticas contables } \\
\text { en ocho países } \\
\text { comunitarios. }\end{array}$ & $\begin{array}{l}\text { Índice I ajustado y el } \\
\text { test del c } 2 \text {. }\end{array}$ & $\begin{array}{l}\text { Los países con las } \\
\text { prácticas contables } \\
\text { más armonizadas son } \\
\text { el Reino Unido y la } \\
\text { Irlanda y los menos } \\
\text { armonizados son } \\
\text { Alemania y Portugal. }\end{array}$ \\
\hline 1996 & $\begin{array}{l}\text { Laínez et } \\
\text { al. }\end{array}$ & $\begin{array}{l}\text { Estudio de los } \\
\text { requisitos de } \\
\text { información exigidos } \\
\text { en las bolsas de } \\
\text { valores. }\end{array}$ & $\begin{array}{l}\text { Índice C de Van } \\
\text { der Tas. Test no } \\
\text { parametritos de } \\
\text { Friedman's y de } \\
\text { Wilcoxon. }\end{array}$ & $\begin{array}{l}\text { Existen diferencias } \\
\text { en la información en } \\
\text { los distintos mercados } \\
\text { financieros. }\end{array}$ \\
\hline
\end{tabular}




\section{Continuación cuadro 2}

\begin{tabular}{|c|c|c|c|c|}
\hline Año & Autores & Alcance & Metodología & Conclusiones \\
\hline 1997 & Krisement & $\begin{array}{l}\text { Estudio de las } \\
\text { prácticas contables } \\
\text { de } 15 \text { países } \\
\text { europeos. }\end{array}$ & $\begin{array}{l}\text { Índice de Entropía, } \\
\text { Índice C y Îndice V. }\end{array}$ & $\begin{array}{l}\text { El índice de entropía es } \\
\text { adecuado para evaluar } \\
\text { la comparabilidad de la } \\
\text { información financiera. }\end{array}$ \\
\hline 1998 & $\begin{array}{l}\text { Weetman } \\
\text { et al. }\end{array}$ & $\begin{array}{l}\text { Estudio de las } \\
\text { prácticas contables } \\
\text { de } 45 \text { empresas } \\
\text { inglesas. }\end{array}$ & $\begin{array}{l}\text { Índice de } \\
\text { conservadurismo de } \\
\text { Gray. }\end{array}$ & $\begin{array}{l}\text { Las empresas cotizadas } \\
\text { están más implicadas } \\
\text { en el proceso de } \\
\text { armonización que las } \\
\text { empresas domésticas. }\end{array}$ \\
\hline 1998 & $\begin{array}{l}\text { Morris y } \\
\text { Parker }\end{array}$ & $\begin{array}{l}\text { Análisis de las } \\
\text { propie-dades } \\
\text { estadísticas de } \\
\text { los índices de } \\
\text { armonización } \\
\text { internacional. }\end{array}$ & $\begin{array}{l}\text { El índice I y el } \\
\text { índice } \\
\text { C entre países. }\end{array}$ & $\begin{array}{l}\text { En las comparaciones } \\
\text { entre más de dos } \\
\text { países, los índices } \\
\text { presentan resultados } \\
\text { distintos. }\end{array}$ \\
\hline 1998 & $\begin{array}{l}\text { Garcia } \\
\text { Benau y } \\
\text { Gandía }\end{array}$ & $\begin{array}{l}\text { Estudio de las } \\
\text { prácticas contables } \\
\text { en } 475 \text { empresas de } \\
\text { nueve países } \\
\text { europeos. }\end{array}$ & $\begin{array}{l}\text { Índice } \mathrm{C} \text { de Van } \\
\text { der Tas y análisis } \\
\text { cluster. }\end{array}$ & $\begin{array}{l}\text { Los países se ubican } \\
\text { en dos grupos, entre } \\
\text { los cuales no existe } \\
\text { armonización. }\end{array}$ \\
\hline 1999 & $\begin{array}{l}\text { Laínez et } \\
\text { al. }\end{array}$ & $\begin{array}{l}\text { Estudio del grado } \\
\text { de armonización } \\
\text { material de prácticas } \\
\text { contables españolas } \\
\text { y del IASB. }\end{array}$ & $\begin{array}{l}\text { Índice C de Van der } \\
\text { Tas corregido: índice } \\
\text { CJL }\end{array}$ & $\begin{array}{l}\text { El grado de armonización } \\
\text { es moderado. }\end{array}$ \\
\hline 1999 & Labrador & $\begin{array}{l}\text { Estudio del grado } \\
\text { de la armonización } \\
\text { contable en España } \\
\text { tras la entrada en } \\
\text { vigor del PGC del } \\
1990 .\end{array}$ & $\begin{array}{l}\text { Descomposición del } \\
\text { índice C de Van der } \\
\text { Tas propuesta por } \\
\text { Archer } \text { et al. }(1995) \text {. }\end{array}$ & $\begin{array}{l}\text { La flexibilidad en los } \\
\text { criterios contables con } \\
\text { lleva a una disminución } \\
\text { de comparabilidad. }\end{array}$ \\
\hline 1999 & $\begin{array}{l}\text { Cañibano y } \\
\text { Mora }\end{array}$ & $\begin{array}{l}\text { Estudio de las } \\
\text { prácticas contables } \\
\text { de } 85 \text { empresas de } \\
13 \text { países distintos. }\end{array}$ & $\begin{array}{l}\text { Descomposición del } \\
\text { índice de C de Archer } \\
\text { et al. Método del } \\
\text { Bootstrapping. Test } \\
\text { del c2 }\end{array}$ & $\begin{array}{l}\text { Existe un proceso } \\
\text { espontáneo de } \\
\text { armonización material } \\
\text { para las global players. }\end{array}$ \\
\hline $\begin{array}{l}2000- \\
2002\end{array}$ & Aisbitt & $\begin{array}{l}\text { Estudio de } 20 \\
\text { prácticas contables } \\
\text { en cuatro países } \\
\text { nórdicos. }\end{array}$ & $\begin{array}{l}\text { La descomposición } \\
\text { del índice C } \\
\text { propuesta por } \\
\text { Archer } \text { et al. }\end{array}$ & $\begin{array}{l}\text { La relación entre la } \\
\text { armonización formal y } \\
\text { la material cambia a lo } \\
\text { largo del tiempo y entre } \\
\text { los países. }\end{array}$ \\
\hline $\begin{array}{l}2000- \\
2002\end{array}$ & $\begin{array}{l}\text { Pierce y } \\
\text { Wetman }\end{array}$ & $\begin{array}{l}\text { Estudio de las } \\
\text { cuentas anuales de } \\
42 \text { empresas } \\
\text { irlandesas y } 125 \\
\text { empresas danesas. }\end{array}$ & $\begin{array}{l}\text { Índice C corregido } \\
\text { con las opciones no } \\
\text { presentadas o no } \\
\text { aplicadas. }\end{array}$ & $\begin{array}{l}\text { El impacto de los } \\
\text { elementos clasificados } \\
\text { como "no presentados" } \\
\text { deberá ser considerado en } \\
\text { el cálculo del índice de } \\
\text { armonización. }\end{array}$ \\
\hline
\end{tabular}




\section{Continuación cuadro 2}

\begin{tabular}{|c|c|c|c|c|}
\hline Año & Autores & Alcance & Metodología & Conclusiones \\
\hline 2002 & $\begin{array}{l}\text { Rahman } \\
\text { et al. }\end{array}$ & $\begin{array}{l}\text { Estudio de las } \\
\text { prácticas contables } \\
\text { de empresas } \\
\text { australianas y Nueva } \\
\text { Zelandesas. }\end{array}$ & $\begin{array}{l}\text { Índice de Jaccard y } \\
\text { Índice de Acuerdo } \\
\text { simple. }\end{array}$ & $\begin{array}{l}\text { La armonización material } \\
\text { está relacionada con la } \\
\text { armonización formal y } \\
\text { con las características de } \\
\text { las empresas. }\end{array}$ \\
\hline 2003 & Jarne et al. & $\begin{array}{l}\text { Análisis de las } \\
\text { prácticas contables } \\
\text { de } 127 \text { empresas } \\
\text { europeas. }\end{array}$ & Análisis porcentual. & $\begin{array}{l}\text { Existe un buen número de } \\
\text { áreas contables donde no } \\
\text { hay uniformidad. }\end{array}$ \\
\hline 2003 & Taplin & $\begin{array}{l}\text { Estimativa del } \\
\text { error estándar de } \\
\text { los índices H y C. } \\
\text { Aplicación a los } \\
\text { datos de Herrmann y } \\
\text { Thomas (1995). }\end{array}$ & $\begin{array}{l}\text { Cálculo del error } \\
\text { padrón. }\end{array}$ & $\begin{array}{l}\text { Las conclusiones de } \\
\text { otros estudios pueden ser } \\
\text { reforzadas o alteradas si } \\
\text { se consideran los errores } \\
\text { padrones en el cálculo del } \\
\text { índice } \mathrm{H} \text {. }\end{array}$ \\
\hline 2004 & Silva & $\begin{array}{l}\text { Armonización de } \\
\text { la información } \\
\text { financiera de } \\
\text { las normas y } \\
\text { prácticas contables } \\
\text { portuguesas. }\end{array}$ & Índice de Jaccard & $\begin{array}{l}\text { La armonización de las } \\
\text { prácticas contables no } \\
\text { depende exclusivamente } \\
\text { de la armonización de las } \\
\text { normas. Quizá futuros } \\
\text { trabajos de investigación } \\
\text { puedan aportar evidencia } \\
\text { empírica adicional. }\end{array}$ \\
\hline 2005 & $\begin{array}{l}\text { Palacios, } \\
\text { Martínez, } \\
\text { Clavel }\end{array}$ & $\begin{array}{l}\text { Factores explicativos } \\
\text { de la diversidad } \\
\text { contable en } \\
\text { Latinoamérica. }\end{array}$ & $\begin{array}{l}\text { Modelo de regresión } \\
\text { lineal }\end{array}$ & $\begin{array}{l}\text { Evidencian que la } \\
\text { comparabilidad está } \\
\text { condicionada por la } \\
\text { normativa contable, } \\
\text { por determinadas } \\
\text { características } \\
\text { empresariales y por } \\
\text { factores coyunturales }\end{array}$ \\
\hline 2009 & Jame et al. & $\begin{array}{l}\text { Prácticas de } \\
\text { valoración y } \\
\text { revelación de } \\
\text { información contable } \\
\text { en Argentina, Brasil } \\
\text { y Chile en el marco } \\
\text { de la armonización } \\
\text { internacional. }\end{array}$ & $\begin{array}{l}\text { El test consiste en } \\
\text { calcular un índice con } \\
\text { la diferencia entre las } \\
\text { variables resultado } \\
\text { y patrimonio neto } \\
\text { considerando los } \\
\text { preparados bajo } \\
\text { US-GAAP y principios } \\
\text { locales de cada país. }\end{array}$ & $\begin{array}{l}\text { Entre los resultados } \\
\text { observamos como los } \\
\text { países de la muestra se } \\
\text { comportan de diferente } \\
\text { manera frente a las } \\
\text { normas norteamericanas, } \\
\text { así como observamos } \\
\text { importantes similitudes } \\
\text { en la revelación efectuada } \\
\text { por estos tres países }\end{array}$ \\
\hline
\end{tabular}

Fuente: Elaboración propia adaptada de Silva (2004). 
Para determinar estas unidades de estudio se tomó en consideración la naturaleza de las operaciones y los criterios señalados por Silva (2004) y Rahman et al. (1996): la importancia de cada elemento para la valoración del patrimonio neto, elementos frecuentemente analizados en otros trabajos y la importancia de los elementos de los estados financieros de las empresas objeto de estudio. En cada una de estas unidades se identificaron un conjunto de atributos, características cuantitativas y cualitativas, producto del estudio realizado a las NIC-NIIF donde se establecen los aspectos más generales e importantes que se deben tomar en consideración para el reconocimiento, medición y revelación de cada unidad de investigación (cuadro 3).

Una vez seleccionadas las unidades de investigación, el siguiente paso fue establecer una medida de proximidad o de distancia entre las prácticas de contabilidad de las empresas objeto de estudio y la normativa establecida por el IASB; asimismo, fue necesario diseñar la empresa modelo que sirvió de comparación.

Las medidas de proximidad, similitud o semejanza miden el grado de semejanza entre dos objetos de forma que, cuanto mayor es su valor, mayor es el grado de similitud existente entre ellos. La medida de proximidad utilizada en el presente estudio es el índice de Jaccard, referenciado por Silva (2004) y que ha sido utilizado en varios estudios sobre armonización contable, basado en datos tipo binario donde las medidas más utilizadas son distancia al cuadrado: $b+c$.

El cálculo de dichos coeficientes se hizo a partir de una tabla 2 X 2 del siguiente tipo:

\begin{tabular}{|l|l|l|l|}
\hline Entes emisores & $\mathbf{1}$ & $\mathbf{0}$ & Total \\
\hline 1 & $\mathrm{a}$ & $\mathrm{b}$ & $\mathrm{a}+\mathrm{b}$ \\
\hline 0 & $\mathrm{c}$ & $\mathrm{d}$ & $\mathrm{c}+\mathrm{d}$ \\
\hline Total & $\mathrm{a}+\mathrm{c}$ & $\mathrm{b}+\mathrm{d}$ & $\mathrm{a}+\mathrm{b}+\mathrm{c}+\mathrm{d}$ \\
\hline
\end{tabular}

El coeficiente de Jaccard está dado por la siguiente expresión $\mathrm{S}=\mathrm{a} / \mathrm{a}+\mathrm{b}+\mathrm{c}+\mathrm{d}$. Donde: $\mathrm{S}=$ Coeficiente de Jaccard, $\mathrm{a}=$ número de pares cuando adopten el mismo tratamiento, $(1,1), b=$ número de pares cuando en la normativa aplicada a las empresas objeto de estudio se trate el tema y en las normativa del IASB no se trate, $(1,0), \mathrm{c}=$ número de pares cuando la normativa aplicada en las empresas objeto de estudio no se trate el tema y en la normativa del IASB se indica un determinado tratamiento 
$(0,1), \mathrm{d}=$ número de pares cuando ambas normativas no traten el tema. En esta investigación se utilizó el sPss (Statistical Product and Service Solutions) versión 15, para obtener el índice de Jaccard.

\section{Cuadro 3}

\section{Unidades de investigación de los elementos de los estados financieros seleccionadas para el estudio empírico}

\begin{tabular}{|c|c|c|c|}
\hline $\begin{array}{l}\text { Número } \\
\text { (UI) }\end{array}$ & NIC/ NIIF & Unidades de Investigación (UI) & $\begin{array}{c}\text { Número } \\
\text { atributos }\end{array}$ \\
\hline $\mathrm{C} 1$ & $\begin{array}{c}\text { NIC- }, 7,8,18,24 \\
33,34\end{array}$ & $\begin{array}{l}\text { Objetivos, características y revelación } \\
\text { de la información financiera }\end{array}$ & 40 \\
\hline $\mathrm{C} 2$ & $\begin{array}{c}\text { NIC- } 2,8,11,12,16 \\
17,18,19,21,23, \\
27,28, \text { NIIF }-5\end{array}$ & $\begin{array}{l}\text { Políticas contables, cambios en las } \\
\text { estimaciones y errores }\end{array}$ & 31 \\
\hline $\mathrm{C} 3$ & nic-2, 11,36, 41 & Inventario & 20 \\
\hline $\mathrm{C} 4$ & $\begin{array}{l}\text { NIC-I } 6,17,2 \mathrm{I}, \\
23,36\end{array}$ & Propiedad, planta y equipos & 20 \\
\hline $\mathrm{C} 5$ & NIC- 38,36 & Intangibles & 14 \\
\hline C6 & $\begin{array}{c}\text { NIC-27, 3I, 32, 33, } \\
36,37,38,39,40 \mathrm{y} \\
\text { NIIF -7 }\end{array}$ & Inversiones financieras & 17 \\
\hline $\mathrm{C} 7$ & NIC-I 2 & Impuesto sobre ganancias & 14 \\
\hline $\mathrm{C} 8$ & nic-37 & Provisiones y contingencias & 15 \\
\hline C9 & NIC-2O & $\begin{array}{l}\text { Contabilización de las subvenciones } \\
\text { del gobierno o información a revelar } \\
\text { sobre ayudas gubernamentales }\end{array}$ & 13 \\
\hline $\mathrm{C} 10$ & NIC-I9 & Planes de pensiones & 10 \\
\hline $\mathrm{C} 11$ & NIC-27, 28, 3I & Inversiones en empresas & 19 \\
\hline $\mathrm{C} 12$ & nic-10 & $\begin{array}{l}\text { Acontecimientos posteriores al cierre } \\
\text { del balance y políticas de reparto de } \\
\text { dividendos }\end{array}$ & 8 \\
\hline
\end{tabular}

Fuente: Gómez y De La Hoz y De La Hoz con base a Silva (2004)

Con respecto a la delimitación de la muestra, se han seleccionado las empresas que cotizan en la Bolsa de Valores de Caracas; se excluyeron las empresas del sector financiero y de seguros, por las particularidades propias de los organismos que las regulan, los cuales exigen la presentación de la información financiera con un plan de cuentas específico.

Una vez delimitadas las empresas objeto de estudio, otro aspecto que se consideró fue la disponibilidad de los estados financieros auditados básicos necesarios para 
obtener los datos, se decidió excluir del análisis a los grupos que no cotizaron en los últimos tres años en forma consecutiva. De un total de 49 empresas que aparecen en el anuario estadísticos de 2007, emitido por la Bolsa de Valores de Caracas, referente al movimiento de registro por título mercado regular, veinte corresponden al sector financiero y seguros y veintinueve al sector no financiero.

Para la presente investigación se han considerado las empresas del sector no financiero, de las cuales sólo fue posible conseguir los estados financieros auditados comparativos de los años 2007-2006 de veinticinco empresas, las cuales se muestran en el anexo 1, y que forman la muestra de la presente investigación.

Una vez establecida la muestra objeto de estudio, se procedió a obtener los estados financieros básicos auditados con sus respectivas notas: balance general, estado de resultados, movimiento del patrimonio neto, estado de flujo del efectivo para los años finalizados en los períodos económicos de los años 2007-2006. Esta información se obtuvo de la página web http://www.cnv.gob.ve/site/index.php de la CNV y de la Bolsa de Valores de Caracas a través de un corredor de bolsa.

Con base en los estados financieros anuales obtenidos se recogieron los atributos para las unidades de investigación; se recolectaron los datos en el cuestionario modelo y se clasificaron en forma dicotómica:

1: Si las empresas reconocen, miden y revelan los elementos de los estados financieros de acuerdo a la norma correspondiente.

0 : Si las empresas no reconocen, miden y revelan los elementos de los estados financieros de acuerdo a la norma correspondiente.

En los casos en que las empresas no presenten la información sobre la norma de contabilidad correspondiente, o no la utilicen, y se evidencie que no se aplica a su caso, se señala con un espacio en blanco; estas situaciones son excluidas del análisis.

\section{Análisis empírico de la armonización contable material}

En el proceso de revisión de los estados financieros auditados de las empresas objeto de estudio se comprobó que las empresas emisoras que cotizan en la Bolsa de Valores de Caracas están sujetas al control de la Comisión Nacional de Valores (CNV), por lo que sus estados financieros se presentan de conformidad con las normas para la elaboración de estados financieros establecidos por dicha Comisión; se indica en las notas de los estados financieros que las normas nacionales no difieren 
significativamente de los principios de contabilidad de aceptación general en Venezuela, emitidos por la FCCPV.

También se observó que no son suficientes los conocimientos desarrollados por la contabilidad financiera para poder integrar la información de los elementos de los estados financieros en cuanto a la medición y revelación. Cuando se utiliza el valor razonable en la medición participan otras disciplinas del saber: valuadores, financieros, actuarios, riesgos, entre otros; en la revelación se requiere un conocimiento integral de la empresa para suministrar a los usuarios toda la información requerida para la toma de decisiones.

Otro elemento observado fue la aplicación de los principios de gobierno corporativo, por estar relacionados con la transparencia de la información financiera, cuyo objetivo es garantizar estándares de transparencia y confianza en los mercados de capitales.

[...] el gobierno corporativo es un sistema de relaciones que involucra al directorio, la dirección ejecutiva, los grupos de control externo e internos y los organismos reguladores para que: aseguren la buena conducta a través de controles y equilibrios, proporcionen control en las decisiones de la gerencia, garanticen la rigurosidad en la documentación de los hechos y creen un ambiente de transparencia [...] Leone (2006).

Según la Resolución No 19-1-2005 de la CNV, se invitó a aplicar los principios de buen gobierno corporativo, dado su reconocimiento en el ámbito local e internacional para alcanzar mercados más confiables y eficientes porque es considerado como un aliado del éxito y el desarrollo sustentable de las empresas en un escenario de competencia global; se observó un movimiento marcado hacia la convergencia de normas de dirección en todo el mundo. A pesar de esta exigencia por parte del ente regulador, solamente el $48 \%$ de las empresas estudiadas manifiestan que cumplen con dichos principios.

En el cuadro 4 se agrupan las empresas de acuerdo con el sector económico, se identifican los estados financieros consolidados o individuales.

Otro aspecto que se detectó es que el $88 \%$ de las empresas mantienen actividades económicas con el mercado internacional bajo diferentes modalidades: bonos al portador, American Depositary Receipt (ADR), inversiones en acciones, clientes, proveedores, instrumentos financieros, entre otros. Asimismo, el 80\% de las em- 
presas son auditadas por las firmas nacionales representantes de las cuatro más grandes del mundo (big four $)^{6}$ y el $20 \%$ representantes de firmas internacionales medianas. También se observó que el $60 \%$ de las empresas pertenecen al sector manufacturero y de la construcción; la poca representatividad de los otros sectores limita la posibilidad de realizar relaciones entre los diferentes sectores y las normas del IASB.

\section{Cuadro 4}

\section{Características de las empresas objeto de estudio}

\begin{tabular}{lllll}
\hline \multirow{2}{*}{$\begin{array}{c}\text { No. } \\
\text { empresas }\end{array}$} & \multicolumn{1}{c}{ Sector económico } & \multirow{2}{*}{$\begin{array}{c}\text { Gobierno } \\
\text { corporativo }\end{array}$} & \multicolumn{2}{c}{ Estados financieros } \\
\cline { 4 - 5 } 3 & Electricidad, gas, agua y & 3 & 2 & 1 \\
\multirow{2}{*}{15} & Servicios & 8 & 14 & 1 \\
3 & Manufacturera y construcción & 8 & 2 & 1 \\
4 & Bienes inmuebles & 0 & 3 & 1 \\
\hline 25 & Agricultura y alimentos & 1 & 21 & 4 \\
\hline
\end{tabular}

Fuente: Gómez, De La Hoz con base en CNV

En relación con el tamaño de las empresas, según el cuadro 5, la mayoría se ubica en menos de 500 millones de bolívares fuertes con leves modificaciones de un año a otro. En cuanto al número de trabajadores no fue posible saberlo porque solamente tres empresas muestran dicha información.

\section{Cuadro 5}

Elementos de los estados financieros

\begin{tabular}{ccccccccc}
\hline $\begin{array}{c}\text { Millones } \\
\text { bolívares fuertes }\end{array}$ & \multicolumn{2}{c}{ Activo } & \multicolumn{2}{c}{ Pasivo } & \multicolumn{2}{c}{$\begin{array}{c}\text { Patrimonio } \\
\text { neto }\end{array}$} & \multicolumn{2}{c}{ Ventas netas } \\
\hline (Bs. F.) & 2007 & 2006 & 2007 & 2006 & 2007 & 2006 & 2007 & 2006 \\
Menos de 500 & 13 & 14 & 18 & 18 & 18 & 18 & 18 & 18 \\
$500-999$ & 5 & 5 & 1 & 1 & 2 & 2 & 2 & 2 \\
$1,000-1,999$ & 1 & 1 & 4 & 4 & 2 & 2 & 3 & 3 \\
Más de 2,000 & 6 & 5 & 2 & 2 & 3 & 3 & 2 & 2 \\
Total & 25 & 25 & 25 & 25 & 25 & 25 & 25 & 25 \\
\hline
\end{tabular}

Fuente: Gómez y De La Hoz con base en los estados financieros.

${ }^{6}$ Deloitte, PricewaterhouseCoopers, KPMG y Ernst \& Young. 
Al observar los resultados presentados en el cuadro 6 se puede decir que para la mayoría de las unidades de investigación estudiadas en más del $70 \%$ coinciden las prácticas de contabilidad utilizadas por las empresas objeto de estudio con relación a las disposiciones establecidas por las NIC-NIIF. Sin embargo, se puede observar que de los 221 atributos examinados un $48 \%$ no aplica a las empresas objeto de estudio o no aparece la información en los elementos de los estados financieros; al aplicar el índice de Jaccard son excluidos de la base de datos para determinar la similitud. Es importante señalar que no se pudieron conocer las razones de esta situación por las siguientes hipótesis: las normas son generales y diseñadas para las grandes empresas donde la complejidad de los negocios puede ser mayor y, por lo tanto, dicho atributo no aplica o falta de información que limita determinar la aplicación o ausencia de la norma.

\section{Cuadro 6}

\section{Agrupación por categorías de estudio}

\begin{tabular}{ccccc}
\hline $\begin{array}{c}\text { Código } \\
\text { (UI) }\end{array}$ & No & Observado & N/A $^{\mathbf{7}}$ & Similitud \\
\cline { 2 - 5 } C1 & 40 & 28 & 12 & 0,739 \\
C2 & 31 & 19 & 12 & 0,936 \\
C3 & 20 & 7 & 13 & 0,915 \\
C4 & 20 & 11 & 9 & 0,879 \\
C5 & 14 & 1 & 13 & 0,962 \\
C6 & 17 & 10 & 7 & 0,871 \\
C7 & 14 & 13 & 1 & 1,000 \\
C8 & 15 & 9 & 6 & 1,000 \\
C9 & 13 & 0 & 13 & N/A \\
C10 & 10 & 1 & 9 & 0,938 \\
C11 & 19 & 9 & 10 & 0,932 \\
C12 & 8 & 6 & 2 & 0,781 \\
\hline Total & $\mathbf{2 2 1}$ & $\mathbf{1 1 4}$ & $\mathbf{1 0 7}$ & \\
\hline
\end{tabular}

Fuente: Elaboración con base en los resultados

La categoría C9 Contabilización de las subvenciones del gobierno o información por revelar sobre ayudas gubernamentales no es una práctica contable aplicada por las empresas que cotizan en la Bolsa de Valores de Caracas porque ninguna

\footnotetext{
${ }^{7}$ No aparece esa práctica de contabilidad en las empresas objeto de estudio.
} 
disfruta de estos beneficios. En la categoría C7 Impuesto sobre ganancias y C8 Provisiones y contingencias, las prácticas contables coinciden en un cien por ciento. Al efectuar una revisión a la base de datos sobre aquellos atributos en los que no existe similitud se evidenció que se refiere a la revelación de información que se debe incluir en las notas a los estados financieros.

En el cuadro 7 se muestran las similitudes para las empresas estudiadas por sector económico, se observa que en el sector electricidad, gas, agua y servicios se presenta una similitud mayor por encima del $80 \%$, dentro de este sector se encuentran dos empresas: la Electricidad de Caracas (EDC) y Compañía Anónima Nacional Teléfonos de Venezuela (CANTV), las cuales fueron líderes en el mercado bursátil venezolano hasta 2006, cambiaron su posición durante 2007 porque según la BVC (2007) se registró una capitalización menor al 70\% en comparación con el cierre de 2006, producto de la nacionalización de ambas.

\section{Cuadro 7 \\ Agrupación por sector económico}

\begin{tabular}{lll}
\hline $\begin{array}{l}\mathrm{N}^{\mathbf{o}} \\
\text { empresas }\end{array}$ & $\begin{array}{l}\text { Sector } \\
\text { económico }\end{array}$ & $\begin{array}{l}\text { Jaccard } \\
\text { Similitudes }\end{array}$ \\
\hline 3 & Electricidad, gas, agua y servicios & 0,842 \\
15 & Manufacturera y construcción & 0,771 \\
3 & Bienes inmuebles & 0,789 \\
4 & Agricultura y alimentos procesados & 0,772 \\
\hline
\end{tabular}

Fuente: Elaboración con base en los resultados

En resumen, se puede decir que existe una armonización significativa entre las prácticas de contabilidad de las empresas estudiadas y las NIC-NIIF de más del $70 \%$, según se muestra en los cuadros 6 y 7 .

\section{Conclusiones}

De acuerdo con la revisión documental realizada, todos los autores coinciden en que el proceso de integración económica internacional ha influido para que la información financiera haya superado las fronteras nacionales, pues su contenido no se limita para los usuarios del país de origen, sino que abarca a usuarios nacionales e internacionales. De esta forma, surge la necesidad de aplicar normas internacionales de información financiera comunes. 
El proceso de convergencia más importante es el desarrollado por el IASB y FASB, quienes cuentan con el apoyo de los reguladores de mercados de capital como la IOSCO, SEC, UE, entre otros. Este proceso se aceleró por el requisito exigido por la Unión Europea (UE) de aplicar las NIC-NIIF a partir de 2005 en los estados financieros consolidados de las compañías que coticen o vayan a cotizar en la UE.

La preocupación de los observadores de este proceso se enfoca en las diferencias existentes entre los fundamentos de ambos modelos de contabilidad. La FASB se ha caracterizado por la emisión de normas que contienen reglas especificas para la orientación en las prácticas de contabilidad; mientras que el IASB ha emitido sus normas sobre la base de un conjunto de principios sólidos suficientes para que los encargados de elaborar la información financiera encuentren siempre las opciones que mejor representen la imagen fiel de la empresa.

Es preciso mencionar que el éxito de la utilización de las NIC-NIIF se debe a que las mismas se fundamentan en principios más que en reglas precisas, donde la sustancia económica y no la forma legal de la transacción es la clave para su reconocimiento, medición y revelación de los elementos de los estados financieros.

El temor que existe en la aplicación de normas basadas en principios es que la discrecionalidad conduzca a lagunas o vacíos que den origen a conductas oportunistas y a la existencia de los fraudes financieros, por lo que el éxito de estas normas dependerá en gran medida del comportamiento ético de quienes son responsables de emitir la información financiera.

Lo anterior indica la necesidad de la búsqueda del conocimiento porque hay signos importantes de cambios en el contexto global que exigen la producción de teórica en la disciplina de la contabilidad. Esto es, romper con una racionalidad meramente operativa y más allá del interés instrumental por trascender a formas de conocer realmente transformadoras y creativas, acordes con las exigencias de la actividad económica-social global, donde se requiere un cambio de paradigma en los empresarios y los profesionales de la contabilidad, quienes junto con los usuarios tendrán que hacer un esfuerzo para entender el marco normativo de las NIC-NIIF.

El proceso de aplicación en Venezuela se inicia parcialmente en 1997 con la aprobación por parte de FCCPV de la DPC-0. Después de más de una década, en 2008, se aprueba el BA VEN NIF N $^{\circ}$ 0, referente al marco de adopción de las NIC-NIIF, el 
cual señala que la aplicación de dichas normas se inicien a partir del 1 de enero de 2008 para las grandes entidades y para las Pyme a partir del 1 de enero de 2011. También se indica que la aplicación de estas normas en Venezuela estará sujeta a la aprobación de la FCCPV.

En cuanto al mercado bursátil en los años 2004 y 2005, la CNV ${ }^{8}$ emitió varias resoluciones referentes a la aplicación de las NIC-NIIF; sin embargo, quedó a potestad de cada empresa aplicarlo anticipadamente; en la actualidad no es obligatorio presentar los estados financieros bajo estas normas.

Al iniciar el estudio empírico con la revisión de los estados financieros se observó que no son suficientes los conocimientos desarrollados por la contabilidad financiera para poder integrar la información de los elementos de los estados financieros en cuanto a la medición y revelación; se requieren conocimientos de otras disciplinas en las áreas de valorización de activos, manejo de instrumentos financieros, actuarios, riesgo, entre otros; en la revelación es necesario un conocimiento integral de la empresa que garantice el suministro de información financiera a los usuarios en general.

Es importante destacar que existe una armonización significativa de más del 70\% entre las prácticas de contabilidad de las empresas estudiadas y las NIC-NIIF; se observaron algunos aspectos comunes como el $88 \%$ de las empresas mantienen actividades económicas con el mercado internacional con diferentes modalidades; el $80 \%$ son auditadas por firmas representantes de las cuatro grandes firmas (big four) de auditoría y el $48 \%$ aplica los principios del buen gobierno corporativo.

Por otra parte, es necesario tener presente que en Venezuela desde 1997 se deben estar aplicando más del 50\% de la NIC- NIIF. Esto más lo indicado en el párrafo anterior justifican los resultados obtenidos; se demuestra que para utilizar estas normas no es necesario que el organismo regulador lo exija, sino la necesidad que tienen las empresas de participar en los mercados internacionales.

En general, se puede decir que la adopción de NIC-NIIF contribuirá a mejorar la percepción internacional de las empresas venezolanas como receptoras de inversiones; es más sencilla la revisión para los inversionistas y cualquier usuario de la información financiera donde los organismos reguladores, gubernamentales, pro-

${ }^{8}$ Para dar cumplimiento a requerimientos de la IOSCO. 
fesionales, emisores de normas de contabilidad y usuario buscan la transparencia y oportunidad de la información financiera.

La aplicación de las NIC- NIIF es un proceso paulatino, que tardará varios años en implantarse y que las mismas están en constante cambio con el propósito de que su aplicación permita reflejar la situación económica-financiera de la entidad, minimizando el grado de incertidumbre.

El presente trabajo servirá de base para nuevas investigaciones que permitan dar aportes a la comunidad científica, docente y profesional sobre el proceso de aplicación de las NIC-NIIF debido a que existe consciencia de que hay que profundizar su estudio y divulgación. A la comunidad científica se le extiende la invitación a participar en investigaciones empíricas en esta área para profundizar su conocimiento.

\section{Referencias}

Amat, Oriol (coordinador) (2005). Comprender las normas internacionales de contabilidad NIC. Barcelona: Gestión 2000.

y Jordi Perramon (2005). Normas internacionales de contabilidad. Barcelona: Gestión 2000.

Bolsa de Valores de Caracas (2007). Anuario Estadístico 2007. Caracas.

Comisión de las Comunidades Europeas (2007). Primer Informe al Comité Europeo de valores y al Parlamento Europeo sobre la convergencia entre las Normas Internacionales de Información Financiera (NIIF) y los Principios Contables Generalmente Aceptados (PCGA) por terceros países. Bruselas. COM405 final.

Federación de Colegio de Contadores Públicos de Venezuela (1974). Normas básicas y Principios de Contabilidad de Aceptación General. Publicación Técnica No 3. Mérida, Venezuela.

(1997). Normas básicas y Principios de Contabilidad de Aceptación General. Declaración de Principio de Contabilidad No 0 (DPC-0). Cabimas, Estado Zulia, Venezuela. 
(2004). Directorio Nacional ampliado de la Federación de Colegio de Contadores Públicos de Venezuela. Araure, Estado Porguesa.

(2004). Directorio Nacional ampliado de la Federación de Colegio de Contadores Públicos de Venezuela. Caracas.

(2007). Marco de Adopción de Normas Internacionales de Información Financiera. BA-VEN- NIF N ${ }^{\circ}$ 0. Caracas, Distrito Capital.

Fundación de Estudios Financieros (2003. Estudio sobre los efectos de la aplicación de las normas contables del IASB a los sectores cotizados de la Bolsa Española. Madrid: Imprime Raro.

Garza Rodríguez, Isabel (2005). China hacia la convergencia con la Normas Internacionales de Información Financiera del IASB. Boletín NIC-NIIF, http://www.nicniif.org, consulta: 20 de marzo de 2007.

Gómez, Francesc (coordinador) (2006). NIC/NIIF. Ejercicios resueltos comentados. Madrid: Gestión 2000.

Gray, S., L. Campabell y J. Shaw (1984). International Financial Reporting: A comparative international survey of accounting requirements and practices in 30 countries. London: Macmillan Publishers.

International Accounting Standards Committee Foundation (2007). Normas Internacionales de Información Financiera (NIIF). United Kingdom: IASF Publications Departament.

Jarne Jarne, José Ignacio, José Antonio Laínez Gadea y Susana Callao Gastón (2003), Grado de convergencia de las prácticas contables de las empresas europeas. Una evidencia empírica. Revista Española de Financiación y Contabilidad. Vol. XXXII (116).

y Fernando Andrés Morales Parada (2009). Prácticas de valoración y revelación de información contable en Argentina, Brasil y Chile en el marco de la armonización internacional. Revista de Estudios Politécnicos. Polytechnical Studies Review, Vol VII (11). 
Krygier, Alberto (1999). Principios de Contabilidad en Venezuela: ¿Qué alcance internacional tendrán? Información financiera: clave para el desarrollo de mercados financieros. Banco Interamericano de Desarrollo

Leone, Carlos (2006). El gobierno de las compañas. Un factor crecientemente crítico. Responsabilidad social Corporativa. Hot Topics. Año 2 (6).

Palacios Manzano, Mercedes, Isabel Martínez Conesa José G. Clavel (2005). Factores explicativos de la diversidad contable en Latinoamérica. Revista Iberoamericana de contabilidad de gestión. (6), pp. 139-160.

Rahman, A., H. Perera y S. Ganesh (2002). Accounting practice harmony, accounting regulation and firm characteristics. Accounting and Business Abacus, Vol. 38 (1), pp. 46-77.

República Bolivariana de Venezuela, Ministerio de Finanzas, Comisión Nacional de Valores. (2004). Resolución N 157-2004. Gaceta Oficial Ordinaria No. 38.085 .

No. 38.354 .

(2005). Resolución $\mathrm{N}^{\circ}$ 177-2005. Gaceta Oficial Ordinarias (2005). Resolución N N 19-1-2005. Gaceta Oficial Ordinarias No. 38.129.

Silva, Ana Fialho (2004). Armonización Internacional de la Información Contable. Análisis y posición de la Normas y Prácticas Portuguesas. Tesis Doctoral. Universidad de Zaragoza. Facultad de Ciencias Económicas Empresariales. Departamento de Contabilidad y Finanzas.

Van der Tas (1988). Measurement harmonization of financial reporting practice. Accounting and Business Research, Vol. 18 (70). 
